\title{
We are on the right road | A brief history of breast cancer guidelines in China
}

\author{
Lili Liao \\ TBCR Editorial Office, AME Publishing Company \\ Correspondence to: Lili Liao. TBCR Editorial Office, AME Publishing Company. Email: tbcr@amegroups.com.
}

Received: 01 July 2021; Accepted: 20 July 2021; Published: 31 July 2021.

doi: 10.21037/tbcr-2021-1

View this article at: https://dx.doi.org/10.21037/tbcr-2021-1

On a spring day in 2006, proposed by Dr. Yan Sun, a famous oncologist in China, the US National Comprehensive Cancer Network (NCCN) guidelines were introduced into China, kicking off the efforts of Chinese scientists and clinicians in developing China's guidelines on tumors.

On another spring day in 2011, with invaluable input and insight from top Chinese oncologists dedicated to advancing cancer care in China, the first Guidelines of the Chinese Society of Clinical Oncology (CSCO) for the Diagnosis and Treatment of Melanoma were published. In April 2021, CSCO had issued 23 clinical guidelines for managing tumors, including breast cancer, colorectal cancer, ovarian cancer, nasopharyngeal cancer, and differentiated thyroid cancer. Other 10 guidelines are also on the way and are scheduled to be published in 2021.

Success is never easy. The 5 -year period from 2006 to 2011 witnessed the efforts and persistence of generations of Chinese oncologists to introduce, adapt, and self-develop clinical practice guidelines for the treatment of tumors. The leadership of our farsighted predecessors and the executive ability of front-line clinicians fighting against tumors are the keys to the success of establishing guidelines with Chinese characteristics. The full respect for science and norms, and the commitment and cohesion of every medical worker in China, have led to several guidelines and updates.

Despite the arduousness, Chinese oncologists are still firmly advancing on this broad road. Among them is Prof. Zefei Jiang (Figure 1), one of the contributors to the NCCN Clinical Practice Guidelines for Breast Cancer. The first Chinese expert voted on the podium of the St Gallen International Consensus Guidelines in Early-stage Breast Cancer, the Chief of the expert group of the CSCO Breast Cancer Guidelines, Vice President and Secretary-General of CSCO,

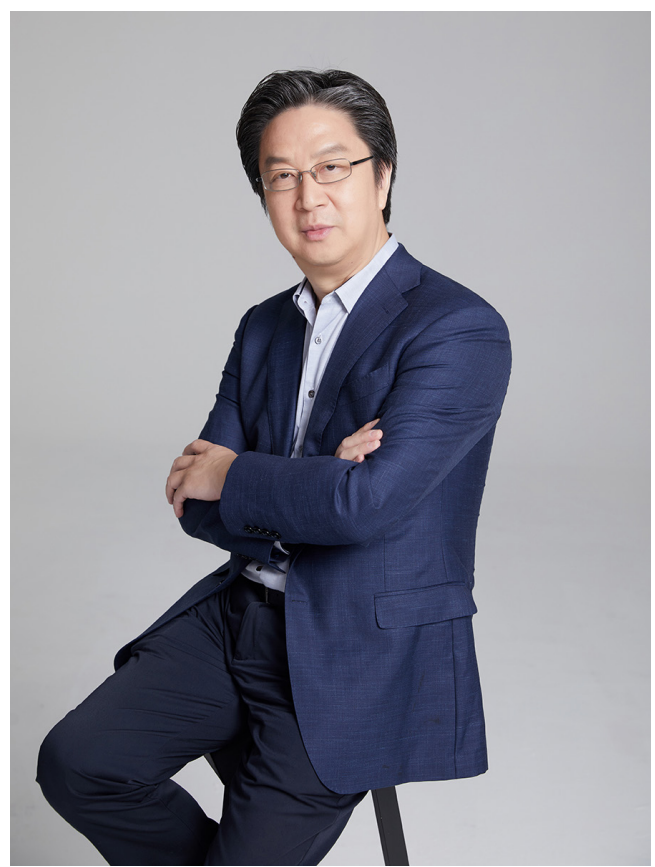

Figure 1 Prof. Zefei Jiang.

and Chairman of the CSCO Breast Cancer Committee.

His career followed the development of China's breast cancer guidelines.

\section{Stones from another mountain can polish jade: the NCCN guidelines}

Zefei Jiang was not surprised by the introduction of the NCCN guidelines into China. He had only returned to China a few years earlier and had maintained close contact with his peers, whom he met in the United States as a 
visiting researcher. During that time, he worked in an institution belonging to one of the NCCN groups, and many of his peers were also members of the expert panels responsible for the development and update of NCCN guidelines.

Zefei Jiang had a strong feeling that "the moment has finally come".

NCCN is a non-profit academic organization made up of 21 major cancer centers in the United States. NCCN Clinical Practice Guidelines in Oncology (NCCN Guidelines) are not only the standards for clinical decisionmaking in oncology in the United States, but have also become widely adopted guidelines worldwide. They have also been recognized and favored by oncologists in China.

The introduction of the NCCN guidelines in 2006 was a milestone. The guidelines, representing the world's leading tumor treatment system, came to China to provide a brandnew benchmark for clinical oncology practice. It was not simply a "translated version", but the "Chinese edition of NCCN Guidelines China", as NCCN officially authorized, and the content could be appropriately adjusted according to Chinese patients' characteristics and medical system. "Thus, the Chinese edition not only benefited tumor patients in mainland China," Zefei Jiang stressed, "but also was helpful for Chinese patients overseas. Although the latter live in other countries, the Chinese edition is more appropriate for them."

For the 39-year-old Zefei Jiang, being one of the contributors to the NCCN Guidelines was still a surprise.

As a contributor, the challenge before him was not merely writing paragraphs or sections but a "young coordinator". The revision of the Chinese edition of these guidelines must correct some traditional habits and consider whether foreign standards were suitable for Chinese clinical reality.

Another challenge was the wide age diversity of the Chinese experts involved in this work. "Some were still 30 or 40 years old, and others were up to 70 or 80 years old." The most important requirement of the contributor was how to coordinate well among these experts. From the beginning, when he received this task from Prof. Yan Sun, Zefei Jiang knew well in his heart that this was the biggest test. However, he also firmly believed, "Since Dr. Sun believes in my ability, I must do it well." After returning to China, Zefei Jiang had attended dozens of academic meetings, large and small, during which Dr. Sun carefully observed his performance. "For Dr. Sun, I just came back from abroad, and English was not a problem for me. And more importantly, be presumed, with my training experience in the United States, that
I would embrace foreign guides, at least easily accept them." Dr. Sun once commented on Zefei Jiang: "he is persistent and not too stupid, or a 'diligent and intelligent person.' Let him do this job as long as he is not exbausted."

These words were comforting for Jiang. He also understood Dr. Sun's ambitions and expectations. "Learn to listen and coordinate well" is a guiding principle for him, and he proved worthy of Dr. Sun's expectations and played a crucial role in coordinating the development of the Chinese edition.

"China implemented a strict family planning policy during those years, and most of the tumor patients in China were middleaged and elderly individuals. Therefore, we removed the topic of 'Fertility Issue in Pregnant Breast Cancer Patients' from the European and American guidelines. In addition, some new drugs licensed abroad may not even be heard of by Chinese oncologists, so how can they be applied?" For such issues with Chinese characteristics, all contributors had to do was to record the deletions and explain the situation to the NCCN truthfully.

There were also a dozen "controversial" issues. For example, in the European and American versions of the guidelines, the dose of a certain drug was $100 \mathrm{mg} / \mathrm{m}^{2}$. Some experts proposed that this dose was intolerable for Chinese patients and should be reduced. "The question arose: What would be a more reasonable dose? 60? 50? You cannot say a figure entirely based on experience and feeling, right? So, the first habit these guidelines trained us was based on evidence. Then the second question came again: the dose of $100 \mathrm{mg} / \mathrm{m}^{2}$ set in the European and American guidelines was based on their population evidence; did we have any evidence for decreasing it? The answer is, unfortunately, no."

The moment Jiang became a contributor, he believed it was a process of seeking common ground while preserving differences. In such a complex system, composed of young, middle-aged, and old oncologists, surgeons, and radiologists, it is possible that sometimes there were dilemmas when "differences" were encountered. "However, all members had a common goal in mind: to contribute to the standardization of breast cancer treatment in China. Voting was our solution to these dilemmas." He was moved that all the panel members, highly respected or up-and-coming stars, followed the principle of majority rule and did not embarrass him too much.

"After the introduction of NCCN guidelines into China, a peak was reached in 2009." Zefei Jiang recalled. At a time when international oncological research was in bloom, these guidelines attracted wide attention. The venues of guidelines-interpreting conferences held in Beijing 
and Shanghai were often full. Long-existing differences arising from different regions, backgrounds, and especially specialties were minimized as much as possible under this common blueprint, and principles in international guidelines have been widely accepted. According to Zefei Jiang, "it was at a time when the traditional model of empirical medicine was evolving into evidence-based medicine, and the NCCN guidelines, to some extent, also changed the way of thinking and practice behaviors of Chinese doctors. That is, the practice of medicine is not individualized behaviors, but regulated behaviors based on scientific evidence." Like other scholars who grew up with the guidelines, Zefei Jiang, who participated in the writing and revision of these guidelines, also gained "incomparable" rewards. "This was an unrepeatable training opportunity. I was fortunate and grateful."

Typically, when one thing reaches its peak and cannot leap to a higher level, it will go from the high to the low point and ultimately vanish. In 2011, 5 years after the publication of the first Chinese edition of the NCCN guidelines, these guidelines regretfully ended due to unconventional conditions, which included differences between the East and West, and the speed of updating the Chinese versions.

"According to the cooperation agreement, NCCN required any additions or deletions to be sent back to them for review before they could be approved. Therefore, after we sent such revisions to NCCN, they had to find an expert who knew Chinese to review it." Each review was time-consuming and seriously affected the speed of the revision. Meanwhile, the NCCN guidelines are often reviewed several times a year due to domestic insurance policies and drug manufacturers' interests. "Sometimes, we had just finished the review of the first edition; the English version had already had the second and even the third editions." In addition to the lag time that naturally occurred, some new drugs and new regimens not yet available in China had also become an insurmountable gap between the Chinese and English versions of these guidelines.

Nevertheless, Zefei Jiang still feels the NCCN guidelines brought us much more than expected. During these five years, with the NCCN guidelines as a starting point, evidence-based medicine was gradually introduced and adopted in China, making a solid step on the road to standardization of cancer diagnosis and treatment in China.

\section{If jade is not polished, it cannot become of use: The St. Gallen Consensus}

In the 5 years since the NCCN guidelines were introduced to China, changes have occurred in the Chinese breast cancer community.

Information sources became more diverse, and more knowledge was learned from other regions, including Europe. The NCCN guidelines themselves had limitations, including limited recommendations in the sections with insufficient evidence and the excessive influence of local policies. Many Chinese breast cancer specialists turned their attention to St. Gallen, Switzerland. The biennial St. Gallen International Breast Cancer Conference and Expert Consensus focuses on the major clinical problems in treating early breast cancer and forms consensuses by voting. It fills in the gaps in guidelines and thus guides clinical treatment choices in a more comprehensive way.

Zefei Jiang began attending the St. Gallen International Breast Cancer Conference early in 2003. Since then, he has maintained his attendance record in all odd-numbered years. Listening to presentations, taking notes, taking photos, and thinking were the routine activities when Jiang attended these conferences. This changed a bit one day in 2010.

Jiang received an email from the organizing committee of the St. Gallen International Breast Cancer Conference and Expert Consensus, which read: "Further, to expand the international influence of the St. Gallen Consensus, we need more voices from different regions and would like to invite you to join us as a representative of the People's Republic of China." In terms of identity, it was a shift from "audience" to "participant". Inwardly, Jiang felt more of a sense of responsibility. In his view, it was not just a simple matter of sitting on the podium, but how to represent China, or even Asia, to make our voice heard.

Zefei Jiang said such responsibility also "greatly boosted his self-confidence". "At a time when the NCCN guidelines were changing quickly, and we were following in a mess, my participation in the development of consensuses recognized by breast cancer specialists from all over the world certainly makes us more confident in the future of breast cancer management in China." In his opinion, the St. Gallen Consensus, which is updated every 2 years, represents a deposit of knowledge from early-stage breast cancer, and there are rarely recommendations canceled and then reincorporated. Instead, these consensuses focus on hot clinical issues and are constantly optimized based on previous ones.

For Jiang, voting is the most "fascinating" link in the development of the St. Gallen Consensus, because "those who are sure to do this do not vote, those who are sure not to do this do not vote, and only those who favor either of the practices 
will vote." Therefore, the issues voted on at the meeting are all the most troubling issues for clinicians. The results of the votes are transparent, and there is no place for pretending to be knowledgeable. Abstentions are allowed during voting. The chairperson may cancel a specific topic, which may be canceled by the chairperson when the abstentions reach a certain percentage to ensure the accuracy and applicability of the voting results. Therefore, "Chinese breast cancer specialists who return with these questions and discussion results can directly apply the conclusions to clinical practice."

More Chinese specialists were on the podium, including Prof. Zhimin Shao from the Fudan University Shanghai Cancer Center in 2013 and Prof. Binghe Xu from the Center Hospital Chinese Academy of Medical Sciences in 2015. The voice of Chinese researchers has been increasingly heard on the international stage. "In the years that we published the Chinese edition of the NCCN guidelines, our opinions could only be delivered to NCCN in text. Today, the situation is different: we can learn about what will be discussed at the meeting three or four months before the meeting starts, and we can also express our opinions in person at the meeting venue." For Chinese scholars, such a change represents a hardearned right to make decisions with strength and effort. "We only bad the right to 'interpret' the NCCN guidelines; however, we have our say in the development of St. Gallen consensuses." Explained Jiang.

Chinese scientists are increasingly speaking out on St. Gallen's podium and in front of the lectern in China. At this moment, this is the opportunity and change that Chinese breast cancer specialists can firmly grasp after years of tireless "polishing".

\section{A piece of jade will be worth thousands of pieces of gold after repeated sculpting: the csco guidelines}

The time was waiting for the birth of the CSCO Breast Cancer Guidelines.

As mentioned above, the NCCN guidelines might be considered a guide from the beginning to the end. The St. Gallen Consensus was a solution to the problems encountered when traffic became burdensome and complex. The CSCO guidelines are updates on the previous iterations, with a better understanding of the user demands and an aim to improve their experience continuously.

The NCCN guidelines and the St. Gallen Consensus represent the characteristics and styles of cancer diagnosis and in the United States and Europe, respectively. Due to many cancer patients and huge ethnic differences in China, both will be poorly applicable if not acclimatized.

"After we have learned the advanced knowledge in foreign guidelines, developing diagnosis and treatment guidelines suitable for Chinese patients based on the specific situations in China has become a top priority and will also reflect the collective wisdom of Chinese specialists." For Zefei Jiang, the availability of the CSCO guidelines can be described as a combination of timing, location, and people. "The timing is perfect, the environment is supportive, and the people have a strong desire." He said.

As more Chinese scientists and clinicians have rich experience in formulating guidelines, and more original clinical studies have been conducted independently in China, the CSCO Council decided to develop and issue our guidelines in 2016. In April 2017, the Chinese Society of Clinical Oncology Breast Cancer (CSCO BC) Guidelines were officially launched. These guidelines adhered to the principles of "evidence-based, accessible, and opinionbased" and took into account regional imbalances, availability, and accessibility of medical resources (especially drugs). The value of oncologic treatment in real-world settings aims to promote standardization of breast cancer management in China.

For Zefei Jiang, the long-awaited moment had finally arrived. "With all the knowledge we have learned, we want to use our products and research to solve our own patients' problems." Chinese specialists now have the autonomy to develop and revise their guidelines. The determination of the top scientists and the resilience of the executors have ensured that the CSCO guidelines travel steadily and far. "Chinese experts, with Chinese wisdom and Chinese guidelines, have solved China's problems," Jiang emphasized.

"Evidence-based medicine is the cornerstone." The selfdeveloped guidelines do not mean that they are made behind closed doors. Instead, high-quality, high-level domestic and international clinical studies remain the most reliable reference in developing guidelines. "Anyway," Zefei Jiang said, "we will consider local health insurance, socioeconomic development, and other circumstances before giving recommendations with Chinese characteristics." For example, accessible and affordable drugs and regimens supported by adequate evidence are unquestionably the basic strategies. However, drugs and regimens that are expensive and poorly accessible in China and moderately effective and affordable to the general population are included as optional strategies.

Applicability is the guiding principle throughout the development of these guidelines. First, the language of the 
guidelines must be concise and easy to understand. Second, for both first- and second-line treatments, the panel sought to put the entire pathway on the same page to make it easier for readers to learn and remember, while those explanatory statements were placed on the next page, "which had not been done in foreign guidelines, but we did." Zefei Jiang said proudly. "In addition, the updates of our guidelines are not as fast as NCCN guidelines, but not as slow as St. Gallen consensuses." Typically, the latest research evidence, opinions and suggestions collected during rounds of feedback from the previous year are summarized in January, during which on-site voting was conducted for key issues; in March, the new version is finalized by the expert panels after rounds of review; and in April, the new guidelines are formally launched. "This timeline balances quality and speed and, therefore, is more reasonable." Said Jiang.

"Feedback is necessary for the panels." A thorough understanding of the subtle needs of our readers adds a new impetus to the change.

"We will also make mistakes. For example, we take it for granted that our readers know what we have written." Zefei Jiang recalled. "One recommendation was that small tumors could be treated with targeted therapy, which we believed our readers knew the prerequisite is negative lymph nodes. Embarrassingly, during the lecture tour, someone asked, 'does it apply in patients with positive lymph nodes?' We realized that the idea we had taken for granted was not understood by all readers and might be misleading. So later, we added 'with negative lymph nodes' in the new version."

In addition to these details, there were even some surprises regarding the format of the recommendations in these guidelines. In the original versions of these guidelines, two or more recommendations were listed as more numerals. Some readers asked, "Does it mean that there is a ranking order for these recommendations?" This reminded the panel. "Change the conjunction to 'or' or use a '.' if the recommendations are not ranked. Use the numerical order only when there is indeed a ranking distinction."

In addition, while updating these guidelines, the "basic strategies and optional strategies" were changed into "grade 1 recommendations, grade 2 recommendations, and level 3 recommendations". Zefei Jiang explained that this adjustment was based on the practice habits of Chinese doctors and the different socioeconomic conditions of the patients. "The grade 1 recommendations represent good efficacy, good accessibility, and wide acceptance; the grade 2 recommendations represent good efficacy but poor accessibility; while the grade 3 recommendations represent fair efficacy but good accessibility, which means, although it is not an optimal protocol, but not a wrong option." These protocols are important guidance for clinicians and may also help drive the health care authorities to approve more indications and urge trial sponsors, such as drug manufacturers, to meet clinical needs further.

The panel also had many new attempts. For instance, they designed the guidelines in a "handbook size" for easy access; or they released a "CSCO AI-assisted diagnosis and treatment tool" and developed a "combined book of guidelines for different cancer types" for consultations. "It feels like another step forward." Jiang smiled.

As the panel leader, the biggest challenge facing Zefei Jiang is how to strike a suitable internal balance when differences arise. As you can imagine, he was sometimes "embarrassed," but he always found a way to resolve the trouble. "Sometimes, it is the views of experienced physicians that contradict international guidelines. Sometimes the trouble comes from pharmaceutical companies, which always strive to put their drugs or protocols in an ideal place. Sometimes the problem is even with me. I am the chief of the panel, but that does not mean that I can do whatever I want," he says. Zefei Jiang's solution is "vote."

However, voting is not a cure-all, and problems still occur from time to time. For example, what percentage of votes can be widely accepted? How to define "acceptable" and how much can be abandoned? Even if a voting result comes, how do you deal with experts who do not accept it? "No way out. It is always hard to reconcile the crowd." Jiang said, "so, I only stick to the principle of the majority rule. In addition to the panel members, junior doctors and interns also bave the right to vote."

Jiang aims to be an impartial organizer of the vote. However, while discussing specific issues, he steps forward to serve as a group leader. "I am the one who comes up with most discussion topics, and if a topic sucks up, it is my problem." Jiang laughed.

"When everyone is willing to belp the team, I believe there is no challenge that we cannot overcome." The road to develop guidelines has no end for the entire Chinese breast cancer community once it is opened. Luckily, more people are gathering on this road.

Today, Zefei Jiang still remembers Dr. Sun's comment, "let bim do this job as long as he is not exhausted." He believes that he remains a persistent and diligent person today, "I want to push some advances in Chinese guidelines and bring something that will benefit our readers."

Sometimes hard work does not always pay off; luckily 
for Zefei Jiang, his efforts in the past years have finally shed light on the road ahead.

\section{Acknowledgments}

I would like to express my heartfelt gratitude to Dr. Zefei Jiang for accepting this interview.

Funding: None.

\section{Footnote}

Provenance and Peer Review: This article was commissioned by the editorial office, Translational Breast Cancer Research. The article did not undergo external peer review.

Conflicts of Interest: The author has completed the ICMJE uniform disclosure form (available at https://dx.doi.

doi: $10.21037 /$ tbcr-2021-1

Cite this article as: Liao L. We are on the right road I A brief history of breast cancer guidelines in China. Transl Breast Cancer Res 2021;2:26. org/10.21037/tbcr-2021-1). The author reports that she is a full-time employee for AME Publishing Company.

Ethical Statement: The author is accountable for all aspects of the work in ensuring that questions related to the accuracy or integrity of any part of the work are appropriately investigated and resolved.

Open Access Statement: This is an Open Access article distributed in accordance with the Creative Commons Attribution-NonCommercial-NoDerivs 4.0 International License (CC BY-NC-ND 4.0), which permits the noncommercial replication and distribution of the article with the strict proviso that no changes or edits are made and the original work is properly cited (including links to both the formal publication through the relevant DOI and the license). See: https://creativecommons.org/licenses/by-nc-nd/4.0/. 